\title{
Improving the Environment for a High-Performance SEM
}

\section{Roland Kappel}

Integrated Dynamics Engineering GmbH, Karl-Liebknecht-Str. 30, 65479 Raunheim, Germany

Roland.Kappel@ideworld.com

\section{Introduction}

Achieving and maintaining high-quality technical performance and research results has been a constant factor in the success of Swerea IVF, the advanced R\&D and consulting service in Molndal, near Goteborg, Sweden. Swerea develops new products, processes, materials, and production technologies through modeling, simulation, testing, and analysis, with a special focus on automated adhesive applications.

When Swerea embarked on an expansion program that included a new research lab in a new location within their complex, they contacted JEOL Skandinaviska AB in Sollentuna, Sweden. Swerea planned to acquire a new instrument-the JEOL JSM-7800F high-resolution analytical field emission scanning electron microscope (SEM) shown in Figure 1. To achieve the top performance from this instrument in the planned location, there was a need for vibration isolation equipment.

Even as Swerea proceeded with the SEM acquisition, vibration isolation expertise was sought from Integrated Dynamics Engineering (IDE) GmbH of Raunheim, Germany. For more than twenty years, Integrated Dynamics has provided environmental solutions including active and passive vibration isolation, EMI cancellation, and acoustic controls. The company was well known to JEOL, the two companies having collaborated on many previous JEOL installations.

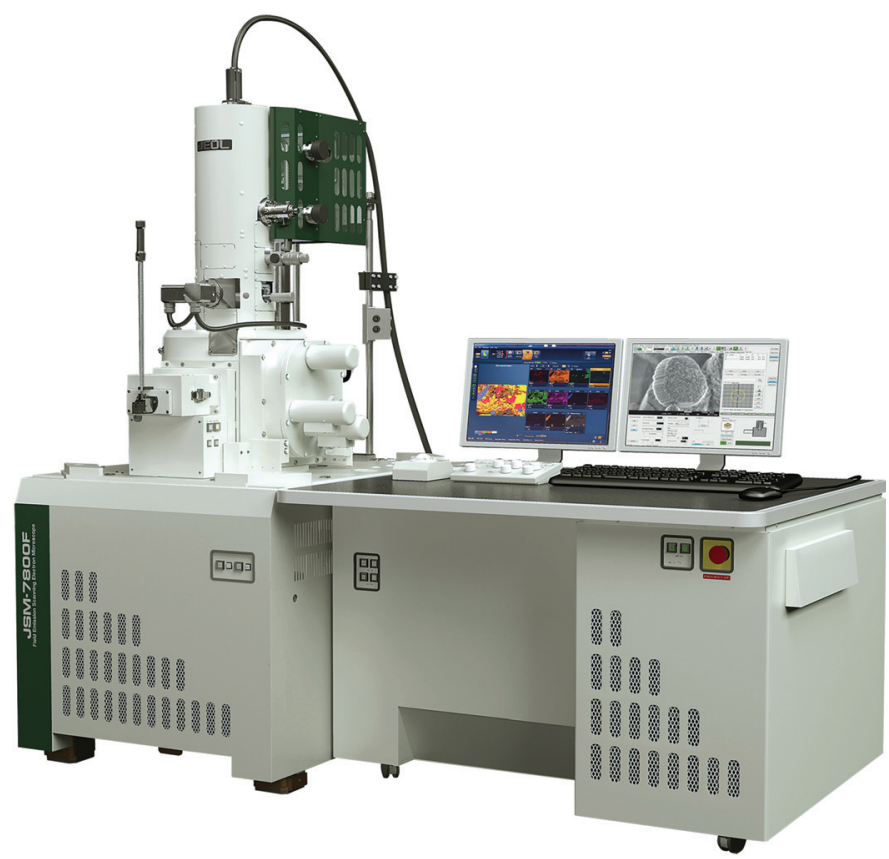

Figure 1: The JEOL JSM-7800F analytical SEM requires low floor vibrations and low electromagnetic fields in order to perform at its highest performance levels. Image courtesy of JEOL USA, Peabody, USA.

\section{Assessing the Environment for a High-Performance SEM}

The high-performance JEOL JMS-7800F SEM was well suited to Swerea's sophisticated research projects, but the new lab location in Molndal had several serious problems. The building was located in a busy section of the downtown area, was anchored in a resonant hard clay bowl, and was adjacent to a heavily trafficked motorway. These were all warning signs that the interior environment of the new Swerea lab would have to be greatly adjusted to accommodate the high sensitivity of the JEOL JSM-7800F, which is capable of imaging nano materials up to $1,000,000 \times$ magnification with sub-nanometer resolution.

Initial tests. The expected interference factors were readily confirmed by JEOL, whose technicians conducted a standard pre-installation site survey in Molndal. The survey revealed an array of abnormally high vibration levels, along with electromagnetic factors in the microscope room. Needing further analysis and definitive solutions to the site problems, JEOL turned to IDE.

IDE site survey. A comprehensive IDE site survey was promptly conducted to ascertain the vibration characteristics and electromagnetic field levels surrounding the proposed microscope location. Floor vibrations were recorded in frequency domain using a fast Fourier transform (FFT) analyzer. Frequency spectra were obtained along three orthogonal axes from $0.1 \mathrm{~Hz}$ to about $500 \mathrm{~Hz}$ [1-2]. Magnetic fieldsweremeasured asDCor ACfields. The DCfield component is the constant or slowly varying part of the total field, with all its energy below $1 \mathrm{~Hz}$. The AC field component is the part of the total field that changes rapidly, with all its energy above $1 \mathrm{~Hz}$.

To achieve the readings JEOL required, IDE employed a high performance laptop with an IDE USB Data Acquisition TANGO unit (including a built-in National Instruments ADC converter). FFT analysis was provided by IDE's proprietary SALSA-Lite V1.0.4 software. Floor vibrations were measured using IDE's unique Biaxis MicroVelocity vibration sensor (BAS-4000). Magnetic fields were measured through a Bartington (Mag-13IE70) Flux Gate Sensor. A Microtech Gefell microphone (Model MK250/ MM210) was used to measure environmental sound pressure levels.

Determining the best location for the instrument. The highway close to the building was the main source of the vibrations detected in the lab. The hard clay ground on which the building was sited also contributed to the vibrations.

Measurements were performed in the first floor laboratory designated to house the SEM. Two possible locations for the SEM within this room were investigated, which are marked 


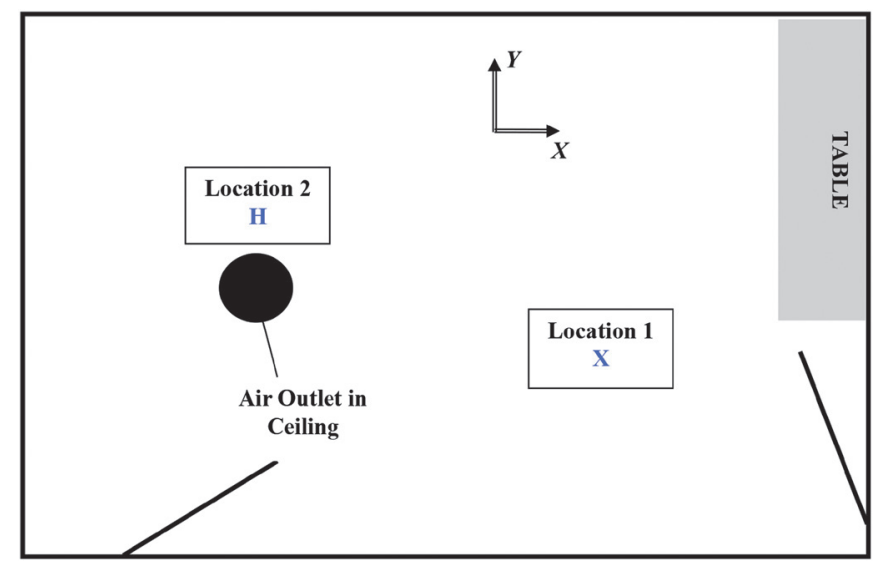

Figure 2: Plan of the SEM room at the Swerea laboratory. The outlet of the air handling system was located in the ceiling close to Location $2(\mathrm{H})$ leading to slightly better acoustical conditions at Location $1(X)$.

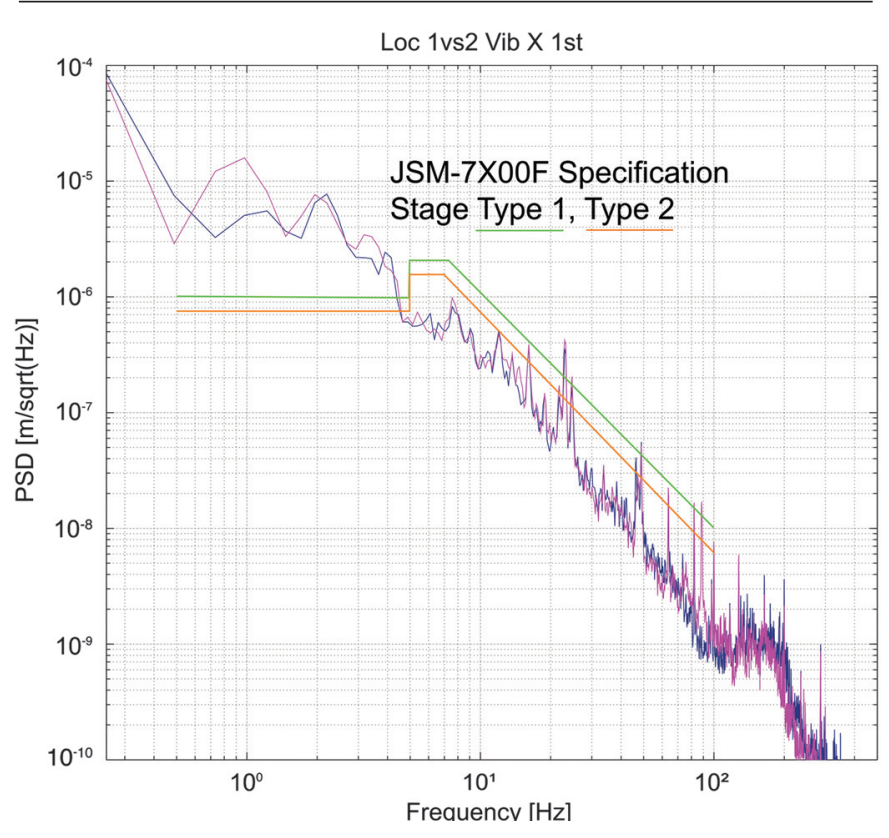

Figure 3: Measurement of floor vibration (displacement in power spectral density (PSD)) in the left-to-right horizontal $X$ direction. Magenta was recorded at Location $1(\mathrm{X})$, and blue was recorded at Location $2(\mathrm{H})$.

with $\mathrm{X}$ (Location 1) and $\mathrm{H}$ (Location 2) in the sketch of Figure 2. Vibration sensors were placed on the floor at the prospective column locations.

There were no known sources for electromagnetic DC fields in the vicinity of the laboratory room. Interference was expected to be mostly from AC fields. The EMI sensor was placed at a height of approximately 1 meter, and readings were taken in three mutually perpendicular axes: $X=$ horizontal (left to right to proposed instrument orientation, refer to sketch), $Y=$ horizontal (front to back to proposed instrument orientation, refer to sketch), and $Z=$ vertical. The EMI measuring devices used DC coupling of the sensors to avoid signal distortions.

\section{Site Survey Results}

Floor vibrations. The detected floor vibration levels registered far above JEOL's specifications. There was a strong horizontal vibration at approximately $1 \mathrm{~Hz}$ reaching

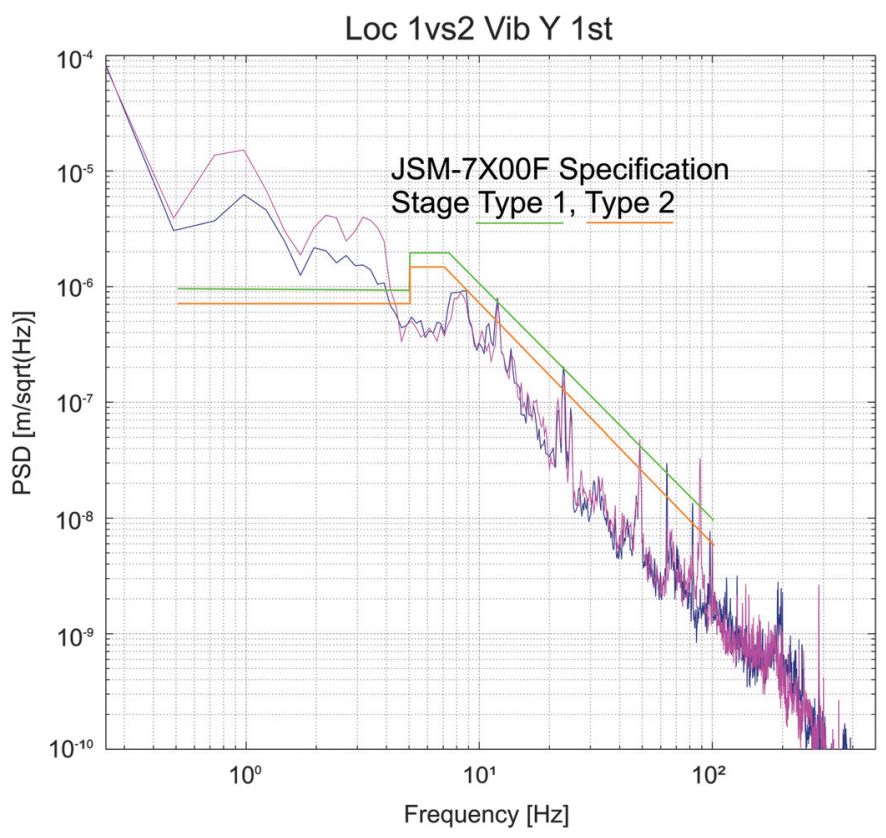

Figure 4: Measurement of floor vibration (displacement in power spectral density (PSD)) in the front-to-back horizontal $Y$ direction. Magenta was recorded at Location $1(\mathrm{X})$, and blue was recorded at Location $2(\mathrm{H})$.

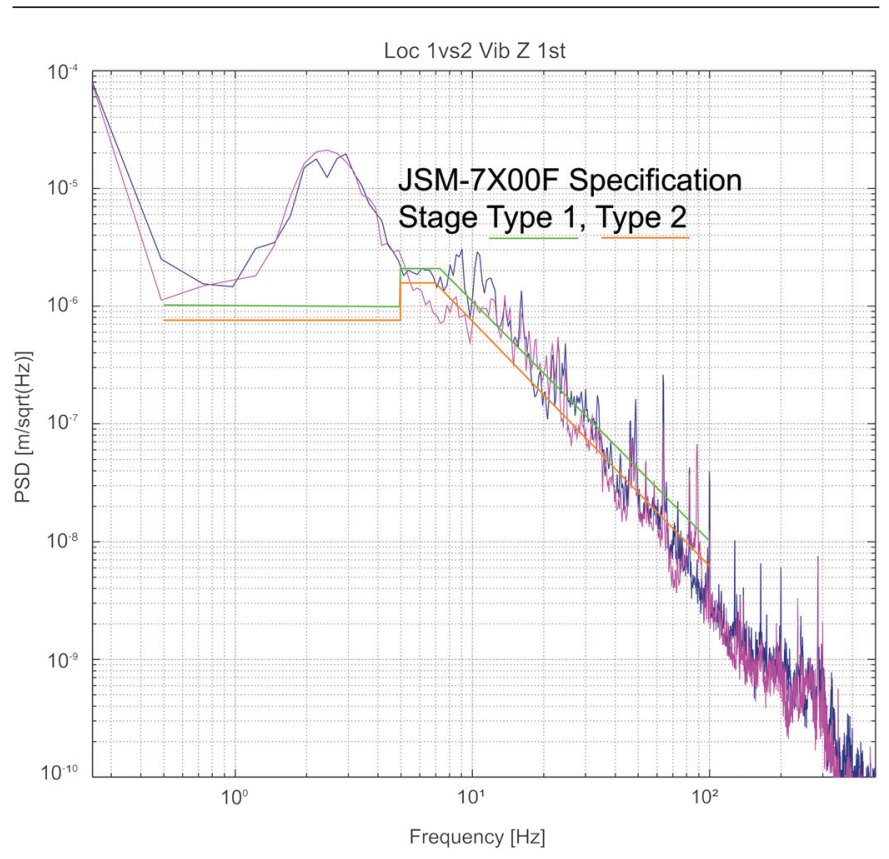

Figure 5: Measurement of floor vibration (displacement in power spectral density (PSD)) in the vertical $Z$ direction. Magenta was recorded at Location 1 $(\mathrm{X})$, and blue was recorded at Location $2(\mathrm{H})$.

up to $15 \mu \mathrm{m} / \sqrt{ }(\mathrm{Hz})$ and up to $20 \mu \mathrm{m} / \sqrt{ }(\mathrm{Hz})$ in the vertical direction at a frequency of $2.3 \mathrm{~Hz}$. Even at high frequencies, the specifications were exceeded, especially in the vertical direction (Figures 3-5). The reason for these strong vibration levels was not entirely clear, but it was likely related to a combination of the building design, the clay ground, and the nearby highway.

The recorded vibration levels were severe. Essentially, there was little difference between Location $1(\mathrm{X})$ and $2(\mathrm{H})$. The levels exceeded JEOL's recommendations by a factor of 20 


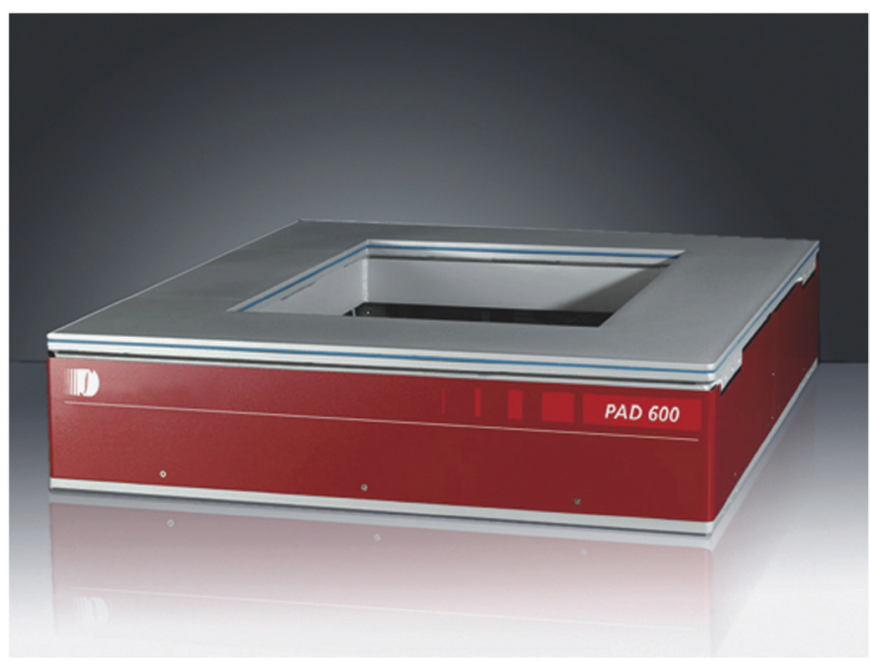

Figure 6: IDE's active vibration platform PAD 600
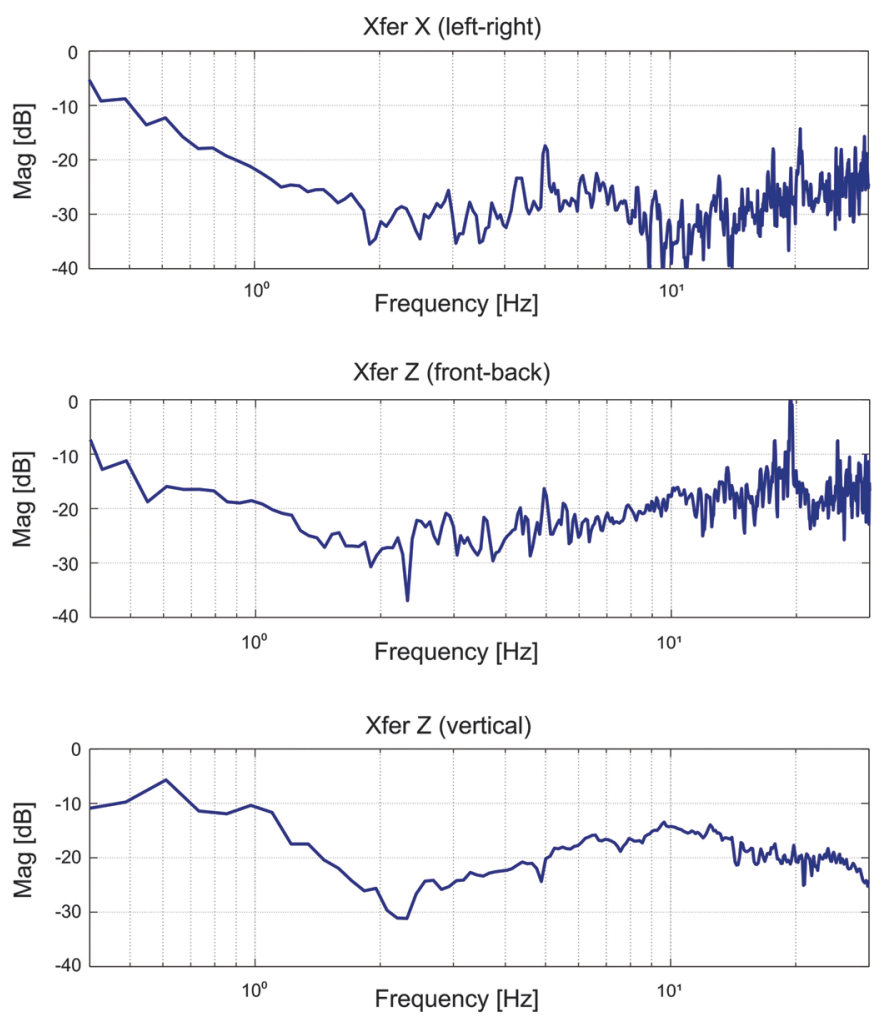

Figure 7: Transfer function diagrams showing attenuation achieved with the PAD 600 active isolation platform. The top graph shows left-to-right $X$ axis, the middle graph shows front-to-back $Y$ axis, and the bottom graph shows vertical $Z$ axis. The blue line shows the positive effects of the PAD 600 with all active control loops working. $0 \mathrm{~dB}$ means $100 \%$ vibration transfer, $-10 \mathrm{~dB}$ means vibration attenuation to $31.6 \%,-20 \mathrm{~dB}$ to $10 \%$, and $-30 \mathrm{~dB}$ to $3.16 \%$ residual vibration.

in the horizontal direction and by a factor of 27 in the vertical direction (with reference to JEOL Stage Type 2 levels).

Electro-magnetic interference (EMI). The recorded EMI exceeded JEOL's recommended maximum of $100 \mathrm{nT}_{\mathrm{pp}}$ for $\mathrm{AC}$ fields at Location 2 (marked with $\mathrm{H}$ in Figure 2). Readings were recorded up to $150 \mathrm{nT}_{\mathrm{pp}}$. Slightly lower readings were measured at Location $1(\mathrm{X})$. Here the maximum reading was $100 \mathrm{nT}_{\mathrm{pp}}$. However, if the JSM-7800F were to be used for low-acceleration voltage operation (low $\mathrm{kV}$ ), the AC field would likely impact the image quality.

The recorded electro-magnetic DC fields met the JEOL specification, except when a chair in the lab had been moved during the measurement. There were no other sources for DC fields identified.

Acoustic interference. Acoustic levels recorded up to $62 \mathrm{~dB}(\mathrm{C})$ using a handheld sound pressure level (SPL) meter. With a class 1 rated microphone, the airflow sound levels at low frequencies ( 8 and $16 \mathrm{~Hz}$ ), caused by the air conditioning system, exceeded the JEOL specification at both locations.

Selected location. The IDE site survey showed that Location 1 (X) exhibited slightly better conditions for the acoustics, because Location $2(\mathrm{H})$ was directly under the air outlet for the lab. Also the AC fields registered slightly lower at Location 1 (X). However, for low-kV operation, an active fieldcancelation system was recommended for both locations.

\section{Recommended Solutions}

Analyzing all the data collected from Swerea site, IDE's testing engineers recommended a course of action to optimize the lab environment. Products marketed by IDE mitigated the identified problems.

Vibration. Replacing the built-in passive JEOL isolation system with an active isolation system would help to reduce vibrations at frequencies below $30 \mathrm{~Hz}$ but would not provide the required reduction at high frequencies. Therefore, an additional isolation system underneath the SEM column was recommended to provide isolation throughout the entire frequency range.

To achieve the required performance, IDE recommended the use of IDE's active vibration isolation platform, the PAD 600 (Figure 6). The PAD 600 provides active vibration isolation with six-degrees-of-freedom to support today's high-performance tools such as this SEM. The PAD 600 employs IDE's Floor Feedforward, a process in which 3-axis floor sensors continuously monitor floor vibrations and feed this information back to a digital controller that processes the sensor signals and effectively reduces low frequency vibrations [3]. This system provides isolation starting at frequencies as low as $1 \mathrm{~Hz}$ - exactly what was needed in Swerea's new laboratory to meet the requirements of the JSM-7800F SEM.

Electro-magnetic interference. Even though the EMI was at or above specification level during the site survey for low-kV usage, with the installation of the PAD 600 the image was not disturbed by magnetic fields. Thus EMI cancelation was not warranted at this time, especially because this SEM would be used primarily at a higher $\mathrm{kV}$ acceleration voltages.

Installation and Tuning. Shortly after the initial IDE site survey, JEOL accepted the IDE recommendations for optimizing the SEM performance with IDE's PAD 600 active isolation system, and installation began. Fine-tuning in this project included IDE's adjustment of the velocity loop parameters and adjustment of the Floor Feedforward parameters. After the first round of tuning, the image was still somewhat distorted, and the distortion appeared to be 
at a frequency around $3 \mathrm{~Hz}$. To correct this, the tuning had to be modified. The attenuation at 2 to $3 \mathrm{~Hz}$ was increased at the cost of the $1 \mathrm{~Hz}$ attenuation. This was clearly a worthwhile trade-off. After that simple adjustment, the image quality greatly improved and is now nearly free from all distortion.

Evidence of Effectiveness. The performance of a vibration isolation system is reflected in the transfer function. Figure 7 shows the magnitude of improvement in the transfer function that is achieved with the PAD 600 at Swerea for the $X, Y$, and $Z$ directions. The $Y$ axis of the transfer function shows vibration magnitude: amplification with positive $\mathrm{dB}$ numbers or attenuation with negative $\mathrm{dB}$ numbers.

Table 1: Attenuation achieved with the PAD 600 active isolation platform

\begin{tabular}{|c|c|c|c|}
\hline \multirow{2}{*}{ Frequency } & \multicolumn{3}{|c|}{ Attenuation in } \\
\cline { 2 - 4 } & X Axis & Y Axis & Z Axis \\
\hline$\sim 1 \mathrm{~Hz}$ & $-21 \mathrm{~dB}$ & $-19 \mathrm{~dB}$ & $-11 \mathrm{~dB}$ \\
\hline$\sim 2.5 \mathrm{~Hz}$ & $-30 \mathrm{~dB}$ & $-25 \mathrm{~dB}$ & $-30 \mathrm{~dB}$ \\
\hline
\end{tabular}
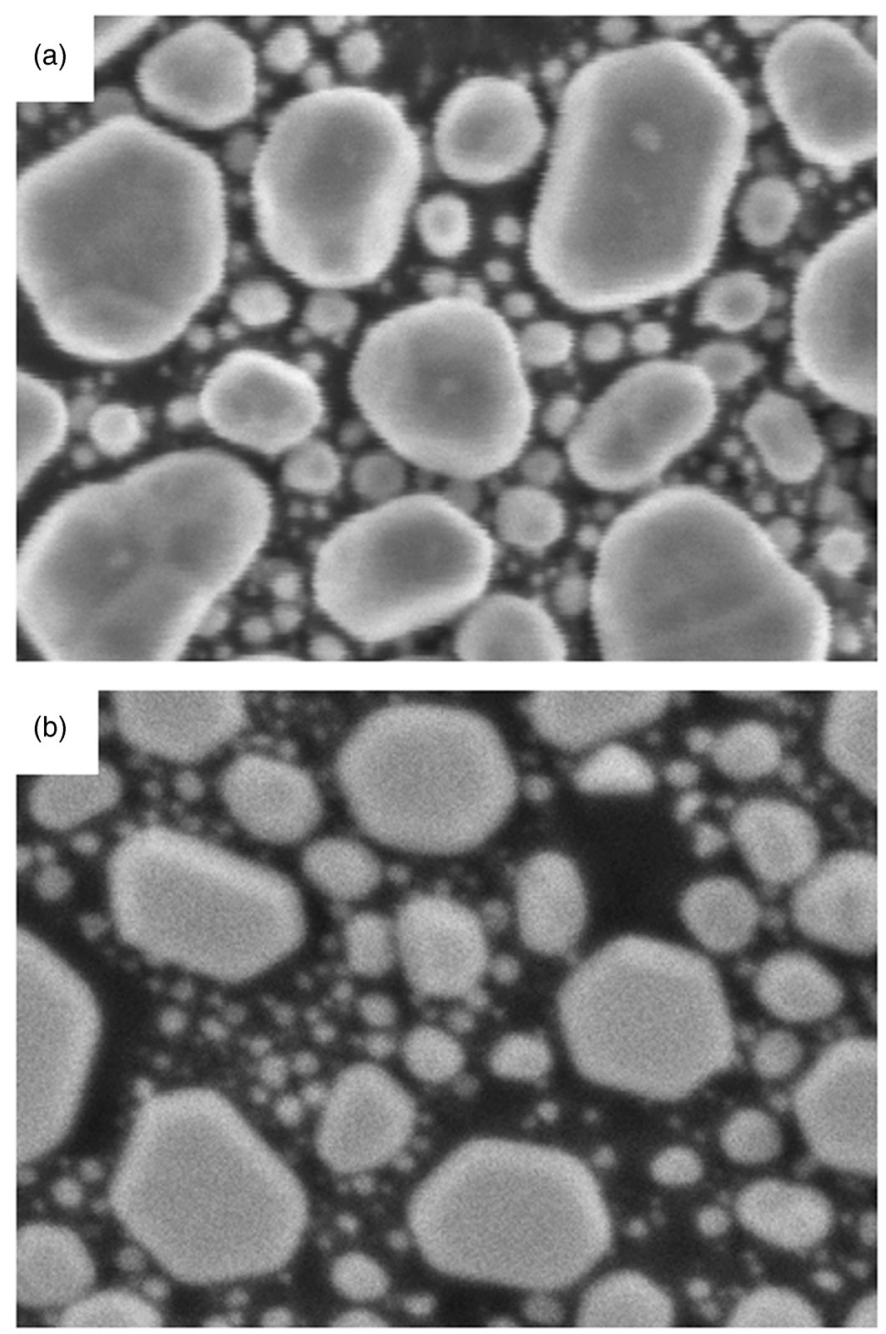

Figure 8: SEM images of a gold-on-carbon test specimen obtained at $1 \mathrm{keV}$ with a working distance of $1.5 \mathrm{~mm}$ (original magnification 130,000x). (a) Image obtained with PAD 600 active isolation platform switched OFF. (b) Image taken with the PAD 600 switched on.
Table 1 shows the level of vibration attenuation. The values in the table indicate the amount of $\mathrm{dB}$ reduction when the PAD 600 is turned on.

The definitive evidence of effectiveness is the quality of the SEM image. Figure 8 shows comparison images of the same area of a gold-on-carbon test specimen without (Figure 8a) and with (Figure 8b) the PAD 600 active vibration isolation platform. In Figure $8 \mathrm{~b}$ note the absence of image tearing visible at the top of Figure $8 \mathrm{a}$. Figure $8 \mathrm{~b}$ shows that all particle edges are sharp and that smaller particles are resolved than would be without the vibration isolation.

\section{Conclusion}

This article describes the details of installation site surveys and provides an example of a successful attenuation of serious vibrations that would have inhibited highresolution SEM imaging. The SEM customer reports that all functions are performing to JEOL's high specification standards, and the research laboratory is obtaining excellent results.

\section{References}

[1] P Maedel, Jr., Vibration Standards and Test Codes, Shock and Vibration Handbook, 5th Edition, ed. C Harris, McGraw Hill Publishing Co., New York, 2001.

[2] H Amick, J Inst Env Sci v. XL, no. 5 (1997) 35-44.

[3] VD Ingenieure, ed., Active Vibration Isolation, VDI 2064 (bilingual German and English), reprinted in VDI-Handbuch Schwingungstechnik, Beuth Verlag GmbH, Berlin, 2010.

\section{Amptek FAST SDD ${ }^{\mathrm{TM}}$ for SEM-EDS Applications}

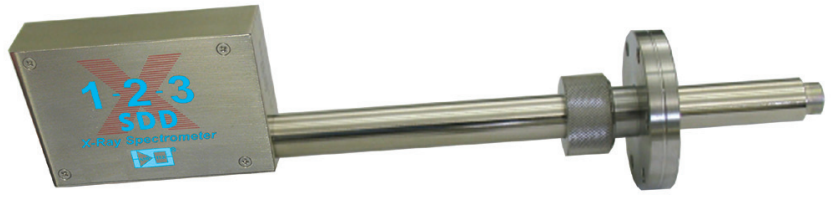

Amptek's new line of SDD detectors for EDS use with SEMs utilizes new technology "C Series" X-ray windows (Si3N4) and has a low energy response down to Carbon. With its high intrinsic efficiency the SDD is ideal for EDS XRF. See why Amptek detectors are the \#1 choice of OEMs worldwide.

\begin{tabular}{|c|c|}
\hline \multicolumn{2}{|c|}{$\begin{array}{c}\text { Transmission for } \\
\text { Low Z Elements }\end{array}$} \\
\hline $\mathrm{B}$ & $19.7 \%$ \\
\hline $\mathrm{C}$ & $43.9 \%$ \\
\hline $\mathrm{N}$ & $59.2 \%$ \\
\hline $\mathrm{O}$ & $62 \%$ \\
\hline $\mathrm{F}$ & $69 \%$ \\
\hline $\mathrm{Ne}$ & $72.9 \%$ \\
\hline $\mathrm{Na}$ & $75.1 \%$ \\
\hline $\mathrm{Mg}$ & $77.3 \%$ \\
\hline $\mathrm{Al}$ & $80.3 \%$ \\
\hline $\mathrm{Si}$ & $81.8 \%$ \\
\hline
\end{tabular}

OEM's \# 1 Choice AMP TEK www.amptek.com 


\section{The SPI Supplies Family} of Instruments

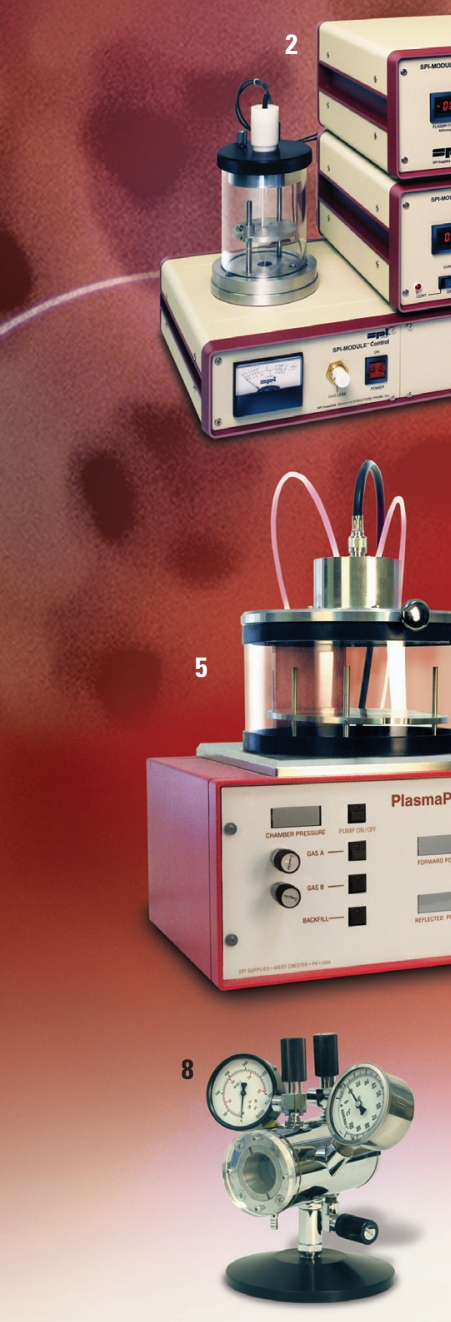

Your results will never be better than

your sample preparation. See how

SPI Supplies can help you deliver the highest quality results for all your SEM/EDS, TEM and FESEM applications.

\section{Excellence in sample preparation just a click away... 2spi.com}

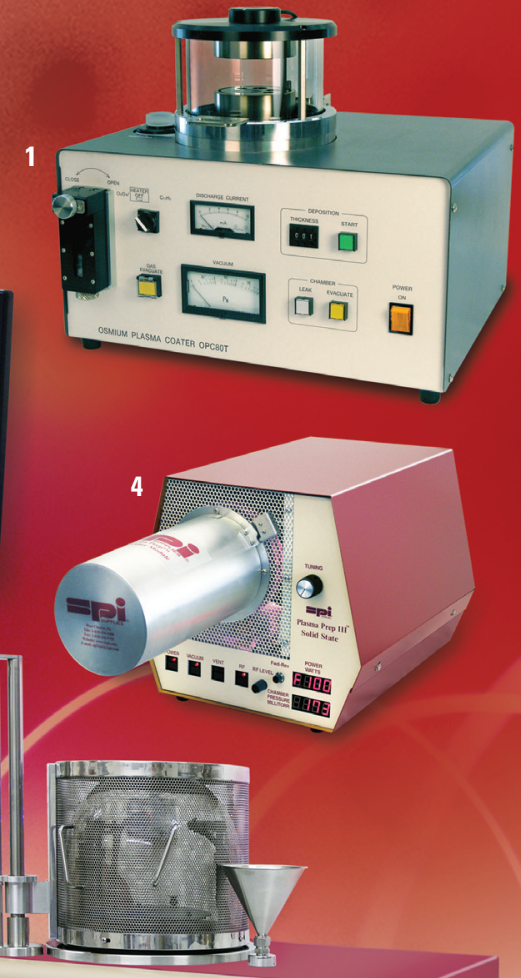

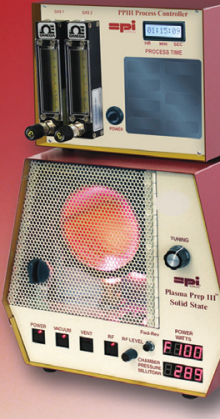

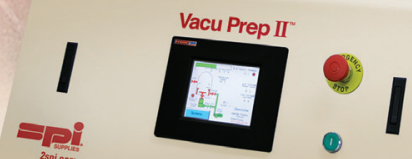

$$
\text { inite }
$$
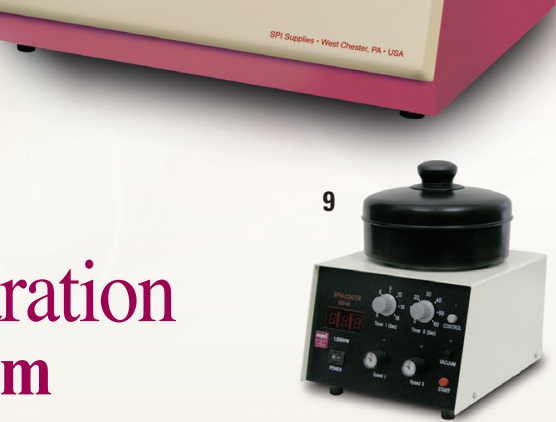

1. Osmium Plasma Coaters for FESEM Applications

2. SPI-MODULE ${ }^{\text {TM }}$

Sputter/Carbon Coater Module 3. Gentle Mill ${ }^{m}$

Ion Milling System

4. Plasma Prep ${ }^{\mathrm{TM}}$ III Solid State

Plasma Cleaner for cleaning TEM holders
5. Plasma Prep ${ }^{\mathrm{Tm}} X$

Parallel Plate Plasma Etcher

6. Plasma Prep ${ }^{\mathrm{rm}}$ III Plasma Etcher with PPIII Process Controller

7. Vacu Prep ${ }^{\mathrm{TM}}$ II

Turbo Pump Evaporation System

8. SPI-DRY'T

Critical Point Dryer

9. Precision Spin Coater Spin coater

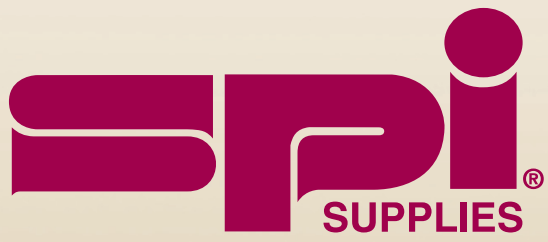

SPI Supplies Division of STRUCTURE PROBE, Inc. 